\title{
Delayed Esophageal Perforation Secondary to Thoracic Aortic Aneurysm Rupture in a Patient with Human Immunodeficiency Virus Infection
}

\author{
Tatsuichiro Seto, MD, PhD, ${ }^{1}$ Tamaki Takano, MD, PhD, ${ }^{1}$ Kazunori Komatsu, MD, ${ }^{1}$ \\ Yoshinori Ohtsu, MD, PhD, ${ }^{1}$ Takamitsu Terasaki, MD, ${ }^{1}$ Yuko Wada, MD, PhD, ${ }^{1}$ \\ Daisuke Fukui, MD, PhD, ${ }^{1}$ Shoichiro Koike, MD, PhD, ${ }^{2}$ and Jun Amano, MD, PhD ${ }^{1}$
}

\begin{abstract}
A 65-year-old man infected with human immunodeficiency virus underwent emergency surgery for rupture of a mycotic descending thoracic aneurysm. The aneurysm was replaced with a prosthetic graft wrapped with omentum. Esophageal perforation occurred 3 weeks after surgery. The patient's condition remained stable, and we adopted a conservative treatment. The esophageal fistula had not healed completely and a biopsy of the scar revealed gastric cancer. We performed a distal gastrectomy, Roux-Y reconstruction, and enterostomy for enteral feeding. Follow-up endoscopy revealed healing of the fistula, and the patient was eventually discharged. We managed this potentially fatal complication with minimally invasive treatment.
\end{abstract}

Keywords: esophageal perforation, aneurysm rupture, human immunodeficiency virus

\section{Introduction}

The incidence of infection by human immunodeficiency virus (HIV) in surgical patients has been increasing in recent years, and these patients develop systemic complications of HIV infection after surgery. In particular, HIV-infected patients have a higher risk of developing postoperative sepsis than do uninfected patients. ${ }^{1-3)}$ As regards cardiovascular surgery, it is reported that procedure for aneurysm is associated with high mortality, ${ }^{1)}$ however surgery with cardiopulmonary bypass seems to be acceptable. ${ }^{4}$ Delayed

${ }^{1}$ Department of Surgery, Shinshu University School of Medicine, Matsumoto, Nagano, Japan

${ }^{2}$ Department of Surgery, Matsumoto Medical Center,

Matsumoto, Nagano, Japan

Received: February 11, 2014; Accepted: May 1, 2014 Corresponding author: Tatsuichiro Seto, MD, PhD. Department of Surgery, Shinshu University School of Medicine, 3-1-1 Asahi, Matsumoto, Nagano 390-8621, Japan

Tel: +81-263-37-2657, Fax: +81-263-37-2721

E-mail: seto@shinshu-u.ac.jp esophageal perforation secondary to thoracic aortic aneurysm rupture is rare, but still remains a fatal complication in spite of improvements in treatment. Therefore, early diagnosis and effective therapeutic management are mandatory. ${ }^{5-8)}$ In case of esophageal resection, a gastric tube is usually used for esophageal reconstruction. Herein, we report a case of esophageal perforation secondary to thoracic aortic aneurysm rupture in a patient with HIV infection and gastric cancer.

\section{Case Report}

A 65-year-old man with a 10-day history of upper abdominal pain and fever was admitted to another hospital. He showed no abnormalities on physical examination with the exception of a low-grade fever. On laboratory examination, the white cell count was $9000 / \mu \mathrm{l}$ and C-reactive protein level was $21 \mathrm{mg} / \mathrm{dl}$. On serological testing, HIV antibody was positive and the CD4 T-cell count was 72 cells/ $\mu$; however, a HIV viral load was not detected. Abdominal X-ray 
showed no abnormalities such as free air; however, abdominal computed tomography (CT) revealed splenomegaly and an unknown lesion around the esophagus. Upper gastrointestinal (GI) endoscopy demonstrated an ulcer scar at the stomach angle, and extrinsic esophageal compression was detected $30 \mathrm{~cm}$ away from the incisors toward the esophagocardial junction. Three days after admission, he developed chest pain. Chest CT showed a 6.5-cm saccular aneurysm of the descending aorta with an irregular aortic wall and a large mediastinal hematoma compressing the esophagus (Fig. 1). Based on the radiological findings, clinical symptoms, and blood examination results, the patient was diagnosed with rupture of a mycotic descending thoracic aneurysm. He was then referred to our hospital, where he underwent an emergent surgery through a left posterolateral thoracotomy. An inflammatory lesion was present around the middle descending aortic aneurysm, and the aneurysm was adhered to the esophagus and lung. No disruption was found in the esophagus at that time. From these findings, the patient was diagnosed with rupture of a mycotic aneurysm, and the aneurysm was replaced with a prosthetic vascular graft wrapped with omentum to prevent infection of the implanted graft. The patient had an uneventful course without infection. However, a low-grade fever, mild leukocytosis, and increased C-reactive protein level developed 3 weeks after surgery. CT showed extraesophageal air and fluid collection in the posterior mediastinum (Fig. 2). Emergency upper GI endoscopy demonstrated mucosal necrosis and perforation of the esophagus to the mediastinal space 35 to $40 \mathrm{~cm}$ from the incisors (Fig. 3). Total parental nutrition was immediately started, and he was maintained with antibiotic coverage. Because his general condition was stable, we decided to perform conservative therapy. Upper GI endoscopy showed improvement in the esophageal necrosis, which decreased in size during the following 2 months. However, the esophageal fistula did not heal completely, and a biopsy of the scar revealed gastric cancer that showed a tendency to increase in size thereafter. He underwent a distal gastrectomy with D2 dissection (preservation of omentum), Roux-Y reconstruction, and enterostomy for enteral feeding. At the sixth postoperative month after the aneurysm surgery, follow-up upper endoscopy revealed healing of the fistula, and he was eventually discharged. He did well after discharge for 5 years,

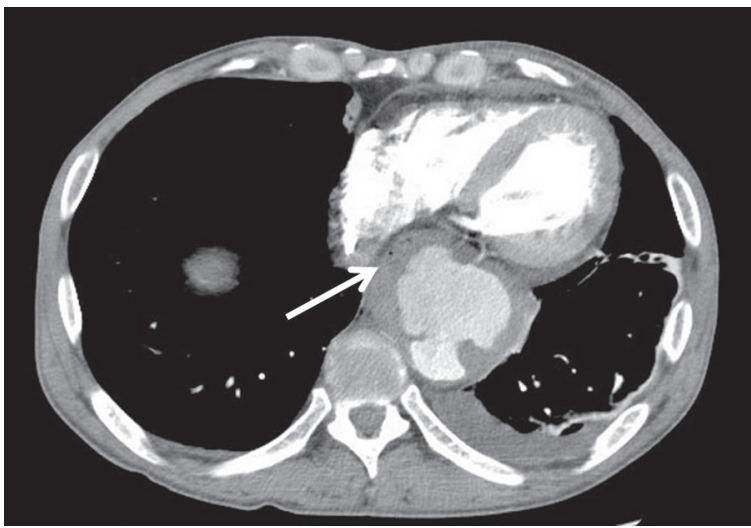

Fig. 1 Chest computed tomography showed a $6.5-\mathrm{cm}$ saccular aneurysm of the descending aorta with an irregular aortic wall and a large mediastinal hematoma compressing the esophagus (white arrow).

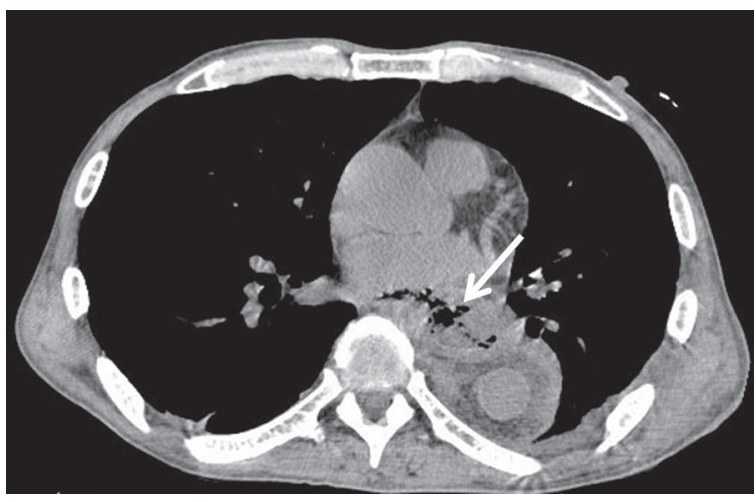

Fig. 2 Chest computed tomography showed extra-esophageal air (white arrow), fluid collection in the posterior mediastinum and a prosthetic vascular graft wrapped with omentum.

but died of pancreatic cancer without evidence of graft infection or recurrence of the gastric cancer.

\section{Discussion}

The number of HIV-infected patients has been increasing in the past decade. Such patients have a higher incidence of postoperative sepsis than do non-HIVinfected patients. ${ }^{1-3)}$ Surgical treatment of HIV-related aneurysms is associated with high perioperative morbidity and mortality. ${ }^{1)}$ The rate of hospital mortality associated with cardiac surgery in HIV-infected patients ranges from $0 \%$ to $40 \%$.9) Surgery with cardiopulmonary bypass does not seem to accelerate the immunodeficiency and is acceptable. ${ }^{4)}$ Because HIV-infected patients are immunocompromised and may have 


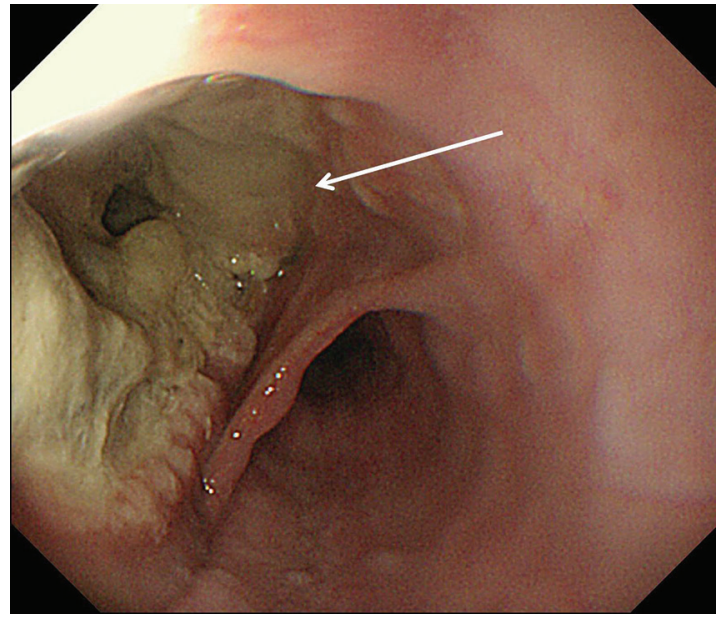

Fig. 3 Upper gastrointestinal endoscopy demonstrated mucosal necrosis and perforation of the esophagus (white arrow) to the mediastinal space 35 to $40 \mathrm{~cm}$ from the incisors.

many complications, these surgical patients should be treated systemically regardless of the surgeon's.

Esophageal disease is a common complication in patients with HIV infection. Esophageal ulceration occurs in up to $30 \%$ of these patients. Ulcers are most commonly caused by cytomegalovirus, and idiopathic ulcers occur almost as frequently. ${ }^{10)}$ Spontaneous esophageal perforation is not common in HIV-infected patients. There have been case reports of esophageal perforation secondary to thoracic aortic aneurysm rupture. The causes of esophageal perforation in patients who undergo surgery for thoracic aortic aneurysm rupture reportedly include arterial occlusion, obstruction of venous return, direct extrinsic esophagus compression, and the graft prosthesis itself. ${ }^{5)}$ In this case, preoperative upper GI endoscopy demonstrated extrinsic esophagus compression with no mucosal lesion. We assumed that the esophageal perforation was mainly caused by a thoracic aortic aneurysm rupture and HIV infection might have lessened the mechanical strength of the esophagus and made it prone to perforation. ${ }^{6)}$

Esophageal perforation is a fatal condition because patients must undergo a second major operation for both the esophageal perforation and prosthetic graft lesion, and this surgery must often be performed under the unfavorable conditions of sepsis. ${ }^{5-8)}$ Low CD4 cell counts, hypoalbuminemia, surgical infection, and major surgery are risk factors for postoperative complications in HIV-infected patients. ${ }^{1-3)}$ This patient had a low CD4 cell count and hypoalbuminemia; furthermore, we considered esophageal resection with colonic reconstruction to be a high-risk procedure. When such a radical procedure seems too risky, conservative treatment should be considered. It is possible to manage this fatal complication with conservative therapy in selected patients without evidence of infection. ${ }^{6-8)}$ This patient's general condition was stable, and the prosthetic graft was covered with omentum. We decided to adopt a conservative strategy. The total hospitalization duration for patients with esophageal perforation undergoing conservative therapy reportedly ranges from 3 to 90 days. ${ }^{7,8)}$ In our case, the esophageal fistula did not heal for a long time. HIV infection may cause esophageal mucosal cell dysfunction, which might delay mucosal cells repair. ${ }^{6)}$

\section{Conclusion}

This is the first reported case of delayed esophageal perforation secondary to thoracic aortic aneurysm rupture complicated with gastric carcinoma in an HIV-infected patient. The selection of minimally invasive treatment for this fatal combination of diseases highlights the importance of careful systemic risk assessment in these complicated patients.

\section{Disclosure Statement}

The authors have no conflicts of interest to declare.

\section{References}

1) Lin PH, Bush RL, Yao Q, et al. Abdominal aortic surgery in patients with human immunodeficiency virus infection. Am J Surg 2004; 188: 690-7.

2) Su J, Tsun A, Zhang L, et al. Preoperative risk factors influencing the incidence of postoperative sepsis in human immunodeficiency virus-infected patients: a retrospective cohort study. World J Surg 2013; 37: 774-9.

3) Deneve JL, Shantha JG, Page AJ, et al. CD4 count is predictive of outcome in HIV-positive patients undergoing abdominal operations. Am J Surg 2010; 200: 694-9; discussion 699-700.

4) Mestres CA, Chuquiure JE, Claramonte X, et al. Longterm results after cardiac surgery in patients infected with the human immunodeficiency virus type-1 (HIV-1). Eur J Cardiothorac Surg 2003; 23: 1007-16; discussion 1016.

5) Park NH, Kim JH, Choi DY, et al. Ischemic esophageal necrosis secondary to traumatic aortic transection. Ann 
Thorac Surg 2004; 78: 2175-8.

6) Serna DL, Vovan TT, Roum JH, et al. Successful nonoperative management of delayed spontaneous esophageal perforation in patients with human immunodeficiency virus. Crit Care Med 2000; 28: 2634-7.

7) Cameron JL, Kieffer RF, Hendrix TR, et al. Selective nonoperative management of contained intrathoracic esophageal disruptions. Ann Thorac Surg 1979; 27: 404-8.

8) Vogel SB, Rout WR, Martin TD, et al. Esophageal perfo- ration in adults: aggressive, conservative treatment lowers morbidity and mortality. Ann Surg 2005; 241: 1016-21; discussion 1021-3.

9) Abad C, Cárdenes MA, Jiménez PC, et al. Cardiac surgery in patients infected with human immunodeficiency virus. Tex Heart Inst J 2000; 27: 356-60.

10) Wilcox CM, Schwartz DA, Clark WS. Esophageal ulceration in human immunodeficiency virus infection. Causes, response to therapy, and long-term outcome. Ann Intern Med 1995; 123: 143-9. 